\title{
Relación entre las dimensiones de personalidad y la presencia de conductas de agresión en adolescentes varones de un colegio particular de Lima Metropolitana.*
}

Relationship between personality dimensions and the presence of aggressive behavior in male teenagers in a private school of Lima Metropolitana.

\author{
Rocío Alexandra Hernández Muro ${ }^{1}$
}

\section{RESUMEN}

El presente estudio tuvo como objetivo principal determinar la relación entre las puntuaciones obtenidas en las dimensiones de personalidad de Eysenck y la presencia de conductas de agresión en 88 adolescentes varones entre 11 a 13 años de un colegio particular de Lima Metropolitana. Para identificar la presencia de las conductas de agresión se elaboró una entrevista estructurada que se aplicó a los tutores de los participantes. A los adolescentes varones se les aplicó el Cuestionario de Personalidad de Eysenck para Niños (EPQ-J). Se obtuvo como resultado principal que existe una fuerte asociación entre la dimensiones psicoticismo y conducta antisocial y la presencia de conductas de agresión. Adicionalmente se obtuvieron los valores de validez y confiabilidad de la prueba EPQ-J y en la entrevista, los cuales resultaron adecuados.

Palabras Clave: Personalidad, agresión, agresión entre pares, EPQ-J, adolescentes varones

\section{ABSTRACT}

The present project had as main objective to determine if there is a significant relation between the obtained punctuation in the dimensions of personality of Eysenck, and to define the presence of aggressive behavior in 88 teenage boys -ages from 11 to 13- of a particular school of Lima city. Eysenck Questionnaire of Personality for Children (EPQ-J) was applied to male teenagers. As a main result, it was obtained that exist a strong association between dimensions of psicoticism and antisocial behaviour and the presence of agression behaviours. Additionally it was found values of validity and confiability in the test, which resulted appropriated.

Key words: Personality, aggression, aggression between peers, EPQ-J, male teenagers.

\section{INTRODUCCIÓN}

Existe un incremento significativo en el interés de estudiar la violencia en contextos escolares, básicamente por los efectos y consecuencias que puede traer a las víctimas de ésta: disminución en la autoestima, trastornos psicopatológicos y compromiso del rendimiento escolar (Fleming, 2002). Sin embargo, es en años recientes que términos como el bullying logran captar la atención de los medios y se incrementan los estudios respecto al tema de agresión asociado a factores como la crianza y la personalidad (Cerezo, 2001).

\footnotetext{
* Investigación ganadora de la "Beca de Estímulo a la Investigación: Barbara Ann kotowski de Tejada" el año 2012, organizado por la UPCH.

1 Egresada de la Facultad de Psicología de la UPCH. Lima-Perú.
} 
La frecuencia de la violencia escolar ha aumentado según los resultados de los estudios (Ohsako, 1997). En Jordania (1996; en Ohsako, 1997) los centros de unión social realizaron un estudio que dio como resultado que en 740 estudiantes el $97.7 \%$ reportó haber presenciado violencia en sus colegios. Asimismo, el récord de ofensas criminales de Malasia (1993; en Ohsako, 1997) indicó que hubo 5290 casos de violencia en la escuela primaria y aumentó a 8658 en la escuela secundaria. El maltrato y la violencia entre alumnos se están convirtiendo en un fenómeno preocupante en las escuelas de América Latina. Según expertos de seis países reunidos en el Panel sobre la Violencia Escolar en Bogotá donde se divulgó una encuesta sobre el tema, se señala que un $22.9 \%$ de los estudiantes es agredido verbalmente por sus compañeros, el $15.6 \%$ objeto de robos o daños en sus pertenencias, un $9.6 \%$ recibe golpes y un $8.2 \%$ es amenazado (Luna, 2007).

La comunidad educativa es el marco donde se desarrolla este tipo de violencia en particular, por lo que todos los integrantes de ésta juegan un rol importante en la forma y frecuencia de la agresión, así como de la prevención e intervención correctiva con el fin de erradicar estas conductas que desvían la atención del objetivo escolar. Tanto los padres de familia como los maestros son entes que median y que pueden detener la violencia entre pares. Quienes están más en contacto con este fenómeno son los profesores o tutores de los alumnos, y son los que dan un primer diagnóstico de este tipo de situaciones. Es por esto que resulta necesario involucrar al maestro, en su rol de autoridad en el aula, en las investigaciones que buscan conocer los factores asociados a la agresión escolar. (Vettenburg, 1999; Smith, Pepler y Rigby, 2004; Miton \& O' Moore, 2004; en Schmidt et al., 2010).

Existen muy pocos estudios e investigación en contextos latinoamericanos, por lo que no se cuenta con muchos datos respecto a la agresión escolar en nuestro país o en realidades similares. Una de las investigaciones revisadas (Mayorga; en Ohsako, 1997) propone que en el Perú los niños se comunican usualmente mediante la agresión y que, inclusive en el núcleo familiar, los padres enseñan a sus hijos a recurrir a la violencia para resolver sus problemas.

Existen algunos estudios realizados en el Perú que indican la frecuencia de agresión escolar en colegios en Lima y el interior del país. Una de las investigaciones realizada por Oliveros, M. et al. (2008) encontró, tanto en provincias como Cusco, Junín, Ayacucho, y en Lima Este, que existe una prevalencia del $47 \%$ en casos de agresión escolar (incluyendo casos de bullying), y que alrededor del $25 \%$ de los maestros no reacciona ni protege a las víctimas permitiendo que este proceso continúe.

Gracias a estos datos, nace la necesidad de realizar estudios que ayuden a identificar la asociación de factores que confluyen en el establecimiento de conductas de agresión. Las investigaciones identifican factores de personalidad asociados a este tipo de conductas; teorías como la de Eysenck han sido de las más eficaces a la hora de determinar dimensiones de personalidad específicas para agresores, víctimas y observadores en la dinámica de agresión entre pares (Ohsako, 1997).

No existen causas determinantes para la agresión, pero sí un conjunto de factores que están asociados a ésta; se podría hablar de una multicausalidad (Universia, 2007; en Olivero et al., 2008). Influyen factores como la escuela, el tipo de educación y la familia que inculca los primeros modelos de comportamiento. Estudios como los de Henggeler et al. (1998; en Espelage \& Sweater, 2003) encontraron que hay una correlación entre el bullying, que es una de las formas que adopta la agresión entre pares, y los altos niveles de conflicto parental y problemas de drogas y encarcelación. Asimismo, los maestros y directores de los colegios pueden ayudar a que se incremente la intimidación si no se establecen políticas que detecten y sancionen el comportamiento agresivo en la escuela (MINEDU, 2006).

La agresión se instaura con mayor frecuencia entre los 11 y los 15 años de edad (Fuligini et al., 2011; en Papalia et al., 2005), etapa de la adolescencia en donde se desarrolla una lucha permanente para establecer los roles que adoptan los sujetos dentro de su espacio social (Pellegrini, 1995; en Pellegrini, Bartini \& Brooks, 1999). Además, se produce una diferenciación en los roles masculino-femenino que afectan la forma y tipo de agresión en los adolescentes que presentan estas conductas (Mandell, 2007). Existen evidencias sobre las diferencias observadas en los tipos de agresión en varones y mujeres; algunas investigaciones afirman que tanto varones como mujeres presentan la misma frecuencia de conductas de agresión (Tamayo, 1999; Ohsako, 1997), otros estudios indican que los varones presentan mayores conductas de agresión que las mujeres; sin embargo, todos los estudios (Duncan, 1999; Melton et al., 1998: Nansel et al., 2011) concluyen que la agresión adopta formas distintas en ambos géneros. 
Los varones optan por formas de agresión más físicas, por lo tanto observables y cuantificables en la mayoría de estudios (Cerezo, Calvo \& Sánchez, 2011). Las mujeres, en cambio, manifiestan mayor agresión relacional; esto significa que se valen de métodos como la coerción, manipulación, exclusión social, con el fin de escalar socialmente. La popularidad en el género femenino está determinado por aspectos de la cultura popular, los cuales marcan las pautas de apariencia física y de conducta; sin embargo, cuando esto no corresponde con las expectativas de apariencia y comportamiento que tiene la niña, puede desencadenar en alteraciones en la autoestima y pasividad ante situaciones de agresión (Mandell, 2006). Asimismo, los roles de víctima - agresor no son estables en el tiempo (como lo es en el caso de los varones), es decir que son dinámicos, y muchas de las víctimas no reconocen estar siendo agredidas porque asumen que son excluidas o ignoradas por una razón válida. Los trastornos alimentarios y la depresión emergen en este contexto (Craig \& Pepler, 2003).

Por estas razones, la violencia en las mujeres durante la adolescencia debe ser tratada de manera diferencial, ya que no existen muchos rastros observables, sino que se instaura de manera indirecta en el desarrollo de los grupos y jerarquías sociales (Cerezo, Calvo \& Sánchez, 2011).

Existen muchas consecuencias para los adolescentes agresores: la posibilidad de inmiscuirse en actividades delictivas, abuso de drogas y alcohol y problemas en el establecimiento de relaciones sociales (Craig \& Pepler, 2003), lo que significan situaciones alarmantes para la sociedad. Sin embargo, las conductas de agresión hacia los pares se pueden prevenir mediante la enseñanza de habilidades sociales y la instauración de políticas de intervención eficaces en los centros educativos (Fernández \& Hernández, 2001).

En la intervención se ha estudiado acerca de la enseñanza de técnicas psicoterapéuticas tanto para las víctimas como para los agresores. Según Espelage, Bosworth, \& Simon (2001, en Forcada et al., 2009) las correlaciones entre rabia y agresión sugieren que el entrenamiento en la regulación y control emocional favorecen la asertividad y reducen la agresión en algunos adolescentes. Sin embargo, aún no existe una capacitación adecuada en los profesionales que están en contacto con los alumnos que les permita identificar señales tempranas que puedan servir de alerta para una atención individualizada (Rodríguez, 2006).
La evaluación de los factores de riesgo es uno de los aspectos que más puede ayudar a la prevención de la conducta agresiva y violenta. Existe un conjunto de variables facilitadoras de la agresión, cuya presencia aumenta la probabilidad de que una persona se comporte de forma agresiva. Existen cuatro aproximaciones (Mossman, 1994; en Carrasco, 2006) en la identificación de sujetos con predisposición a cometer actos de agresión: mediante la evaluación de experiencias previas de violencia, mediante la evaluación de la amenaza y sus vías de proceso hasta que se convierte en agresión, a través del despistaje de problemas de conducta asociado a factores de riesgo (acceso a armas, apoyo familiar) y mediante listados de conductas denominadas como agresivas.

Factores como la personalidad, la educación y el entorno social y cultural determinan el tipo de agresión y su frecuencia, lo cual se ha observado en múltiples estudios en los últimos años (Henggeler et al., 1988; en Espelage \& Sweater, 2003; Cerezo, 2002). Sin embargo, existen pocas referencias acerca del papel que tienen los docentes como entes de atención primaria en los problemas de agresión (Rigby, 2002; en Serrano, 2006). Los maestros son generalmente los primeros en detectar las conductas de agresión, especialmente los que asumen el rol de tutoría y pasan mayor tiempo con un grupo de alumnos; por lo cual resulta necesario involucrar el papel del docente en los estudios que se realizan sobre la violencia en el contexto escolar (Cerezo, Calvo \& Sánchez, 2011; Serrano, 2006).

Más allá de identificar aquellos factores que hacen que la agresión sea más frecuente, como las condiciones del centro educativo (permisividad o carencias de autoridad de los maestros), la crianza de los padres y las condiciones socioculturales (Henggeler, Schoenwald, Borduin, Rowland \& Cunningham, 1998; en Espelage \& Sweater, 2003), existen estudios que refieren que hay una tendencia clara en la personalidad de ciertos alumnos que los hace proclives a usar la agresión como medio para lograr sus objetivos sociales (Olweus, 1998; en Hernández \& Sevilla, 2006; Tomeek, 1995, en Pellegrini, Bartini \& Brooks, 1999; Ohsako, 1997).

En este marco de referencia nace la inquietud de conocer si existe una asociación entre las dimensiones de personalidad y la presencia de conductas de agresión en adolescentes varones, lo cual nos permite formular la siguiente pregunta de investigación:

¿Cuál es la relación entre las dimensiones de 
personalidad y la presencia de conductas de agresión en adolescentes varones de un colegio particular de Lima Metropolitana?.

\section{OBJETIVO}

Determinar la relación entre las dimensiones de personalidad y la presencia de conductas de agresión en adolescentes varones de un colegio particular de Lima Metropolitana.

\section{HIPÓTESIS}

Existe relación significativa entre las dimensiones de personalidad y la presencia de conductas de agresión en adolescentes varones de un colegio particular de Lima Metropolitana.

\section{MÉTODO}

\section{Nivel y Tipo de Investigación.}

El nivel de la investigación es básica debido a que busca la generación de conocimientos sin modificar la realidad. El tipo de investigación es descriptiva, no experimental ya que no se realiza ninguna manipulación intencional de variables (Alarcón, 1991).

\section{Diseño de la Investigación}

La investigación sigue un diseño correlacional, ya que se busca determinar si las variaciones en los valores de una variable se encuentran acompañados de variaciones en la otra variable.

\section{Participantes}

La muestra accesible estuvo constituida por 88 alumnos varones de 11 a 13 años, que cursaban el primer y segundo año de educación secundaria de un colegio particular de Lima Metropolitana.

\section{Instrumentos}

Se aplicó el Cuestionario de Personalidad para Niños de Eysenck (EPQ-J) que mide 3 dimensiones de personalidad: neuroticismo, extraversión y psicoticismo. Además se aplicó una escala de sinceridad y de conducta antisocial. Este Cuestionario está validado en nuestro país en varios estudios, sin embargo, para esta investigación se utilizó la versión de Mori (2002).

También se aplicó una entrevista estructura a los tutores de los alumnos para conocer la presencia o no de conductas de agresión. Para determinar la validez y confiabilidad del instrumento se realizaron dos tipos de análisis: la validez de contenido mediante el criterio de jueces y el análisis de confiabilidad que dio un Alfa de Cronbach de 0.892 que según Oviedo y Arias (2005) es alta.

\section{Procedimiento}

Luego de conseguir los permisos pertinentes de la institución educativa donde se ejecutó la investigación, se aplicaron, tanto la prueba de personalidad y el instrumento para la entrevista, a alumnos y profesores respectivamente.

Se realizaron los análisis estadísticos del caso y se presentaron los resultados, discusión y conclusiones.

\section{RESULTADOS}

En la tabla $\mathrm{N}^{\circ} 1$ se observa la correlación obtenida entre la dimensión neuroticismo y la presencia de conductas de agresión (0.023) la cual es baja.

En la tabla $\mathrm{N}^{\circ} 2$ se muestra la correlación obtenida en la dimensión Extraversión y conductas de agresión, esta fue baja (-0.017).

Tabla $N^{\circ} 1$ : Neuroticismo y Conductas de Agresión

\begin{tabular}{llc}
\hline & & $\begin{array}{c}\text { Conductas } \\
\text { de Agresión }\end{array}$ \\
\hline Neuroticismo & Correlación de Pearson & .023 \\
& Sig. (bilateral) & .840 \\
& $\mathrm{~N}$ & 88 \\
\hline
\end{tabular}

Tabla N²: Extraversión y Conductas de Agresión

\begin{tabular}{llc}
\hline & & Extraversión \\
\hline Conductas & Correlación de & -.017 \\
Agresión & Pearson & .880 \\
& Sig. (bilateral) & 88
\end{tabular}

Tabla Nº3: Psicoticismo y Conductas de Agresión

\begin{tabular}{llc}
\hline & & Psicoticismo \\
\hline $\begin{array}{l}\text { Conductas } \\
\text { Agresión }\end{array}$ & Correlación de Pearson & .617 \\
& Sig. (bilateral) & .000 \\
\hline & $\mathrm{N}$ & 88
\end{tabular}

Tabla Nº4: Sinceridad y Conductas de Agresión

\begin{tabular}{|c|c|c|}
\hline & & Sinceridad \\
\hline \multirow[t]{2}{*}{$\begin{array}{l}\text { Conductas } \\
\text { Agresión }\end{array}$} & Correlación de Pearson & 193 \\
\hline & Sig. (bilateral) & .086 \\
\hline
\end{tabular}


Tabla N5: Conducta Antisocial y Conductas de Agresión.

\begin{tabular}{llc}
\hline & & $\begin{array}{c}\text { Conducta } \\
\text { Antisocial }\end{array}$ \\
\hline $\begin{array}{l}\text { Conducta } \\
\text { Agresión }\end{array}$ & Correlación de Pearson & .565 \\
& Sig. (bilateral) & .000 \\
\hline & $\mathrm{N}$ & 88
\end{tabular}

Tabla $N^{\circ}$ 6: Estadísticos Descriptivos

\begin{tabular}{lccc}
\hline & $\mathrm{N}$ & Media & $\begin{array}{c}\text { Desviación } \\
\text { Típica }\end{array}$ \\
\hline Neuroticismo & 88 & 9.977 & 4.264 \\
Extraversión & 88 & 17.761 & 3.447 \\
Psicoticismo & 88 & 4.102 & 2.537 \\
Sinceridad & 88 & 12.284 & 4.201 \\
Conducta & & & \\
Antisocial & 88 & 19.397 & 4.159 \\
\hline $\mathrm{N}$ & & &
\end{tabular}

Tabla No 7: Niveles de Neuroticismo

\begin{tabular}{lll}
\hline & $\mathrm{f}$ & $\%$ \\
\hline Alto & 13 & 14.77 \\
Promedio & 46 & 52.27 \\
Bajo & 29 & 32.95 \\
\hline $\mathrm{n}=$ & 88 & 100
\end{tabular}

En la tabla $\mathrm{N}^{\circ} 3$ se observa que la correlación entre psicoticismo y conductas de agresión es alta (0.617).

En la tabla $\mathrm{N}^{\circ} 4$ se presentan los resultados de la correlación entre sinceridad y conductas de agresión, esta correlación fue de 0.193 (baja).

En la tabla $\mathrm{N}^{\circ} 5$ se tiene que la correlación entre Conducta antisocial y conductas de agresión es alta (0.565).

A continuación, en la tabla 6 se presentan los estadísticos descriptivos. La media del puntaje de neuroticismo es 9.99 (con una desviación estándar de 4.3), para extraversión es 17.76 (con una desviación estándar de 3.4), para psicoticismo es 4.10 (con una desviación estándar de 2.5), para sinceridad es 12.28 (con una desviación estándar de 4.2) y para conducta antisocial es de 19.40 (con una desviación estándar de 4.2).
Tabla $N^{\circ}$ 8: Niveles de Extraversión

\begin{tabular}{lll}
\hline & $\mathrm{f}$ & $\%$ \\
\hline Alto & 13 & 14.77 \\
Promedio & 57 & 64.77 \\
Bajo & 18 & 20.45 \\
\hline $\mathrm{n}=$ & 88 & 100
\end{tabular}

Tabla No 9: Niveles de Psicoticismo

\begin{tabular}{lll}
\hline & $\mathrm{f}$ & $\%$ \\
\hline Alto & 36 & 40.91 \\
Promedio & 49 & 55.68 \\
Bajo & 3 & 3.41 \\
\hline $\mathrm{n}=$ & 88 & 100
\end{tabular}

Tabla N’ 10: Niveles de Sinceridad

\begin{tabular}{lll}
\hline & $\mathrm{f}$ & $\%$ \\
\hline Alto & 47 & 53.41 \\
Promedio & 41 & 46.59 \\
Bajo & 0 & 0.00 \\
\hline $\mathrm{n}=$ & 88 & 100
\end{tabular}

Tabla N 11: Niveles de Conducta Antisocial

\begin{tabular}{lll}
\hline & $\mathrm{f}$ & $\%$ \\
\hline Alto & 26 & 29.55 \\
Promedio & 55 & 62.50 \\
Bajo & 7 & 7.95 \\
\hline $\mathrm{n}=$ & 88 & 100
\end{tabular}

Tabla $N^{\circ}$ 12: Regresión Lineal

\begin{tabular}{cccccc}
\hline & $\begin{array}{c}\text { Coeficientes no } \\
\text { estandarizados }\end{array}$ & $\begin{array}{c}\text { Coeficientes } \\
\text { estandarizados }\end{array}$ & t & Sig. \\
\hline & B & $\begin{array}{c}\text { Error } \\
\text { típ. }\end{array}$ & Beta & B & $\begin{array}{c}\text { Error } \\
\text { típ. }\end{array}$ \\
& 2.863 & 3.326 & & 0.861 & 0.392 \\
N & -0.208 & 0.225 & -0.183 & -0.926 & 0.357 \\
E & -0.017 & 0.289 & -0.012 & -0.057 & 0.955 \\
P & 0.765 & 0.303 & 0.465 & 2.529 & 0.013 \\
S & -0.165 & 0.161 & -0.146 & -1.027 & 0.308 \\
CA & 0.071 & 0.354 & 0.064 & 0.202 & 0.841 \\
\hline Variable
\end{tabular}

Variable: Conductas de agresión

En la tabla $\mathrm{N}^{\circ} 7$, se observa los resultados obtenidos por los participantes para la dimensión neuroticismo. El $52.27 \%$ se encuentra en el nivel promedio, mientras que el $32.95 \%$ se ubica en un nivel bajo y el restante $14.77 \%$ en un nivel alto. 
En la dimensión extraversión (tabla 8) también se encuentra que en su mayoría los participantes se ubican en una categoría promedio $(64.77 \%)$, seguido de un $20.45 \%$ en nivel bajo y $14.77 \%$ en nivel alto.

Para la dimensión psicoticismo (tabla 9) se observa algunas variaciones en los resultados. La mayoría de la muestra se ubicó en un nivel promedio (55.68\%); sin embargo, existe un $40.91 \%$ en el nivel alto y solo $3.41 \%$ en nivel bajo.

Asimismo, en la dimensión sinceridad, (tabla 10) no existen participantes que obtengan un nivel bajo, la mayoría $(53.41 \%)$ se encuentra en un nivel alto, y el restante $46.59 \%$ en un nivel promedio.

Finalmente, para los niveles de la conducta antisocial (tabla 11) se identificó a un $62.5 \%$ en nivel promedio, $29.55 \%$ en nivel alto y solo un $7.95 \%$ en nivel bajo.

Además, se realizó una regresión múltiple usando como referencia la variable conductas de agresión. Se utilizó como variables predictoras las dimensiones de personalidad. En la tabla 12 se observa que la dimensión con mayor poder predictivo es psicoticismo con un coeficiente beta de 0.465 .

\section{DISCUSIÓN}

Previo al tratamiento de los resultados se realizó una prueba de normalidad mediante el uso del estadístico Kolmogorov-Smirnov. Se encontró que todas las variables alcanzaron una distribución normal, tanto las de personalidad como la variable conductas de agresión, por lo tanto, se llevó a cabo el procedimiento de las correlaciones entre estas.

Los resultados muestran que la dimensión con mayor fuerza de asociación a la variable conductas de agresión, es la dimensión psicoticismo, la cual obtiene un coeficiente Pearson de 0.617, valor que según Martínez et al. (2009) indica una asociación alta; asimismo, la dimensión conducta antisocial correlaciona con conductas de agresión con un valor Pearson de 0.565 , que también resulta alto. El resto de variables obtiene valores menores o iguales a 0.193 lo cual indica una baja asociación entre estas y la presencia de conductas de agresión.

Desde la inclusión de la variable psicoticismo al modelo psicobiológico de la personalidad de Eysenck (1987; en Mori, 2002) se le vislumbró como una variable independiente del neuroticismo y extraversión, que indica la tendencia a la impulsividad, la baja empatía y la agresividad hacia otras personas.

Si bien en puntajes muy altos se asocia con una tendencia a la psicosis (Eysenck, 1970), en puntajes medios-altos se asocia a conductas de carácter antisocial y criminal, lo cual se observa tanto en la relación obtenida con psicoticismo, como con la dimensión conducta antisocial. Esta última dimensión fue elaborada con ítems del resto de dimensiones, que se ha observado predisponen a los sujetos a cometer actos etiquetados como agresivos o delincuenciales (Staub, 1999; en Connoly \& O’ Moore, 2002).

Además, se halló resultados similares con lo encontrado por López y López (2003), en cuyo estudio se asoció las variables de personalidad de Eysenck con la conducta antisocial y delictiva. Sus resultados muestran una asociación de 0.417 para psicoticismo y conducta antisocial, el cual resulta moderadamente alto. En los resultados expuestos en esta investigación la asociación es aún mayor, probablemente debido a que se consideró solo el grupo de varones, ya que otros autores revisados indican que la mayoría de incidentes de violencia se reportan entre adolescentes de sexo masculino (Ohsako, 1997; Nansel et al., 2001; Olweus, 1993; Bulton \& Unverwood, 1992; en Fleming, 2002). Una probable explicación podría estar dada por la influencia androgénica (Tamayo, 1999) o el aprendizaje de modelos agresivos desde la cultura (Bandura, 1983; en Chertok, 2009). Sin embargo, otros estudios indican que no existen diferencias en la frecuencia de la agresión entre varones y mujeres, pero sí diferencias en el tipo de agresión que ejercen sobre sus pares (Craig \& Pepler, 2003; Olweus, 1992; en Fleming, 2002). La investigación realizada por FierroHernández (2000) también concluye que la dimensión psicoticismo sobresale con puntajes más altos en varones que en mujeres.

Siguiendo con el análisis de los resultados obtenidos, existen investigaciones que corroboran la asociación entre las variables de personalidad propuestas por Eysenck y la presencia de conductas de agresión. Cerezo (2001) encontró en su investigación comparativa entre alumnos agresores y víctimas, que los agresores tenían puntuaciones altas en psicoticismo, y que a su vez, esta dimensión era discriminante entre ambos grupos. Asimismo, Connolly y O'Moore (2002) en su estudio de personalidad, utilizaron el EPQ-J $\mathrm{y}$ encontraron que los agresores mostraban mayor inhibición emocional, lo cual es uno de los rasgos 
que caracterizan a las personas con alto psicoticismo, acorde a lo propuesto por Eysenck (1990; en Schmidt, V. et al., 2010). Hernández y Sevilla (2006) realizaron un estudio que les permitió elaborar un perfil de adolescentes con conductas de agresión; algunos de sus resultados señalan que los alumnos agresores tienen alta necesidad de dominar a los demás, pobre autocontrol, sinceridad y alto psicoticismo, entre otras características.

Una investigación realizada en la Universidad de Puerto Rico (Porrata et al., 2011) evaluó la personalidad usando el Cuestionario de Eysenck (EPQ-J) en adolescentes identificados como agresivos. El estudio mostró que los alumnos que eran detectados como agresores puntuaban más alto en las dimensiones psicoticismo y sinceridad.

Finalmente, otro estudio español asocia la variable psicoticismo a adolescentes que son identificados como antisociales dentro de su grupo de pares. En este estudio realizado por Alvarez (2009) las variables que predispone a un adolescente a ser considerado como agresor son las dimensiones psicoticismo y conducta antisocial, cuyos niveles son significativamente más altos en dicho grupo comparado al resto de adolescentes; resultados similares se encontró en la presente investigación.

Se encontraron valores que permiten describir a los participantes según el puntaje obtenido en las dimensiones de personalidad. Se identificó en primera instancia los estadísticos de tendencia central, como la media y la desviación estándar. Al respecto, los resultados se aproximan mucho a los obtenidos por Lemos et al. (1992) en España y a Mori (2002) en nuestro país, en su muestra de varones. Asimismo, los datos para la desviación estándar en ambos estudios se encuentran entre 2.5 (psicoticismo) y 4.2 (neuroticismo).

En la presente investigación la mayoría de la muestra presentan puntajes de nivel promedio y bajo en la dimensión neuroticismo ( $85 \%$ se ubica en ambos niveles), lo mismo ocurre con la dimensión extraversión, ya que el $85 \%$ obtiene nivel promedio o bajo; esto concuerda con lo encontrado en la revisión bibliográfica, ya que los niveles más altos en neuroticismo los obtienen en su mayoría muestras femeninas; sin embargo, en extraversión los puntajes son estables en ambos sexos (Moya \& Meseguer, 2010). En la dimensión psicoticismo sí se halló una tendencia hacia los puntajes más altos, $56 \%$ se ubica en nivel promedio y un $41 \%$ de los participantes se ubica en nivel alto; esto también se observa en la dimensión sinceridad, ya que ningún participante obtuvo nivel bajo; en cambio, el 53\% se ubicó en un nivel alto.

Como se describió anteriormente, existe una clara tendencia en los varones a presentar puntajes significativamente altos en psicoticismo y sinceridad en la evaluación de las dimensiones de personalidad (Lemos et al., 1992). Para la dimensión conducta antisocial, los puntajes tienden más hacia el promedio $(62.5 \%)$.

Los resultados obtenidos en la regresión múltiple permiten determinar el poder predictivo de las variables examinadas. En este caso, la fuerza predictiva mayor que se identificó fue de beta 0.465 para la dimensión psicoticismo. La investigación de López \& López (2003) llega a la misma conclusión usando estadísticos predictivos para las variables de personalidad.

Estos resultados pueden ser importantes en la medida en que se puede utilizar esta variable (el medio-alto puntaje en psicoticismo) como factor de riesgo en las evaluaciones que se realizan a los alumnos de los centros educativos, para identificar problemas o posibles problemas de agresión. Si bien existen otros factores determinantes (Tamayo, 1999), dentro de la teoría de Eysenck, los sujetos con puntajes medianamente altos presentan comportamiento agresivo e impulsivo, entre otras características (Eysenck \& Eysenck, 1987 en Mori, 2002). Hay que tomar en cuenta que esta teoría no busca ser determinista sino que explora la confluencia de aspectos aprendidos y aspectos heredados para la expresión de rasgos estables en la personalidad. Tanto el aprendizaje, situaciones sociales hostiles y factores del temperamento contribuyen en las conductas de agresión. Este concepto habla de un factor general que predispone a la psicopatía, conducta criminal y antisocial y, en mayor grado, al comportamiento psicótico. Sobre este último punto existen diversas investigaciones que relacionan las conductas agresivas en los adolescentes con altos niveles de conflicto parental, problemas de drogas y encarcelación en la juventud (Olweus, 1980 y 1993; en Espelage \& Sweater, 2003).

Otro aporte de este estudio se centra en la importancia de rescatar el papel como observador para el diagnóstico de situaciones de agresión escolar. La fuerte asociación encontrada entre la medida psicológica (psicoticismo) y el criterio del tutor, valida 
y refuerza la idea de involucrarlos mucho más en los procesos de prevención e intervención de violencia en las aulas (Teruel, 2007).

\section{CONCLUSIONES}

1. La correlación entre la presencia de conductas de agresión y neuroticismo, extraversión y sinceridad, es baja (0.023, 0.017 y 0.193 , respectivamente); sin embargo, existe una asociación alta para la dimensión conducta antisocial, de 0.565 y para la dimensión psicoticismo, de 0.617 .

2. Respecto a las medidas de tendencia central, se obtuvo una media de 9.98 para la dimensión neuroticismo, una media de 17.76 para extraversión, 4.10 para psicoticismo, 12.28 para sinceridad $\mathrm{y}$, finalmente, la media de la dimensión conducta antisocial es de 19.40 .

3. Se realizó una regresión lineal múltiple para identificar la dimensión de personalidad con mayor fuerza predictora. Entre los índices encontrados, la dimensión psicoticismo resulta la mayor predictora para la presencia de conductas de agresión, ya que obtiene un beta de 0.465 .

\section{REFERENCIAS BIBLIOGRÁFICAS}

1. Andrés-Pueyo, A. et al. (2005). Evaluación y medida de la agresión, la agresividad y la violencia. Universidad de Barcelona. Proyecto de Investigación del Ministerio de Ciencia y Tecnología del Gobierno de España.

2. Carrasco, M. (2006). Evaluación de la conducta agresiva. revista acción psicológica 4 (2) 76-83.

3. Cerezo, F (2002). La agresión entre pares y su relación con las actitudes de socialización en una muestra de adolescentes. Recuperado de http://66.102. $1.104 / \mathrm{scholar} ? \mathrm{hl}=\mathrm{es} \& \mathrm{r}=\& \mathrm{q}=\mathrm{c}$ a che $: Z \mathrm{~B}$ sePgr21 f8J:dialnet.unirioja.es/servlet/articulo $\% 3 \mathrm{Fco}$ digo\%3D1031387\%26orden\%3D29408\%26info\%3 Dlink+liderazgo+en+adolescentes.

4. Cerezo, F (2001). Variables de personalidad asociadas en la dinámica agresión entre pares (agresores versus víctimas) en niños y niñas de 10 a 15 años. Recuperado de http://www.um.es/facpsi/analesps/v17/v17_1/0417_1.pdf

5. Cerezo, F., Calvo, A. \& Sanchez, C. (2011). Programa CIP: Intervención psicoeducativa y tratamiento diferenciado en el agresión entre pares. Madrid: Pirámide.

6. Cerezo, F. (2002). Bull- S. Test de evaluación de la agresividad entre escolares. Madrid: Albor-Cohs.
7. Chang, G. (2011). En torno a la agresión. Revista Athenea 2 (1) 45 - 60. Recuperado de http://www. academiaperuanadepsicologia.org/athenea $\% 202 / 50$. htm

8. Chertok, A. (2009). Desarrollo y tratamiento de los comportamientos agresivos. Enfoque Cognitivo-Conductual. Conferencia dictada en la Sociedad de Psiquiatría de Uruguay, Montevideo. Extraída de http://www.spu.org. uy/ DesarrolloyTratamientodeComportamientos Agresivos.pdf Connolly, I. \& O'Moore, M. (2002) Personality and family relations of children who bully. Recuperado de http://www.sciencedirect.com/ science?_ob=ArticleURL\&_udi=B6V9F-479TDCT$4 \&$ _user $=10 \&$ _coverDate $=08 \% 2 \mathrm{~F} 31 \% 2 \mathrm{~F} 2003 \&$ $\mathrm{rdoc}=1 \& \_$fmt $=$summary\&_orig=browse\&_sort $=\mathrm{d} \&$ view $=c \& \_$acct $=C 000050221 \&$ \&ersion $=1 \& \_u r l$ 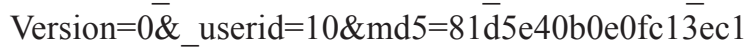 86ff5682daae34

9. Craig \& Pepler (2003). Identifying and targeting risk for involvement in aggression entre pares and victimization. Can J Psychiatry, 48 (9) 54-63.

10. Craig \& Pepler (1998). The relationship among aggression, bullying victimization, depression, anxiety, and aggression in elementary school children. Personality and Individual Differences, 24 (2), 123130.

11. Daft, R. (2006). La experiencia del liderazgo. México: Thompson.

13. Delgado, H. (1966). La personalidad y el carácter. Lima: Centro Editorial Universidad Peruana Cayetano Heredia.

14. Espelage, D. (2004). La intimidación en el comienzo de la adolescencia: La función del grupo social. Recuperado de http://www.ericdigests.org/2003-4/ grupo.html

15. Estarelles, R., Gracia, E., García, F. \& Preito, J. (1989). Variables predictoras de la conducta antisocial. Revista de Psicología Universitas Tarraconenses. Vol XI (1) España.

16. Espelage, D. L., Holt, M. K., \& Henkel, R. R. (2003). Examination of peer group contextual effects on aggressive behavior during early adolescence. Child Development, 74 (1), 205-220.

17. Eysenck, H. (1970). Fundamentos biológicos de la personalidad. Barcelona: Editorial Fontanella.

18. Fernández. \& Hernández (2001) El Maltrato entre escolares. Guía para Jóvenes. Madrid: Fondo Editorial de la Defensoría del Menor.

19. Fierro-Hernández, C. (2000). Patrón de rasgos personales y comportamiento escolar en jóvenes. Facultad de Psicología de la Universidad de Málaga, Revista de Educación 332 (1) 76-83.

20. Fleming, M. (2002, Mayo). Youth bullying. Foro Educacional en Salud Adolescente. American Medical Association, Chicago. 
21. Forcada, R., Pardo, N. \& Bondía, B. (2006). Impulsividad en dependientes de cocaína que abandonan el consumo. Recuperado de http://www. adicciones.es/files/111-118\%20FORCADA.pdf

22. Forcada, R. et al. (2009). Perfiles delictivos, sentimiento de íra y tipo de violencia en adolescentes con grave historial judicial. Revista de la Asociación Española de Neuropsiquiatría, 104 (29) 75-84.

23. Goleman, D. (1998). La inteligencia emocional. Bueno Aires: Javier Vergara Editor.

24. Hernández, M. \& Sevilla, C. (2006). El perfil del alumno agresor en la escuela. Trabajo presentado en el Congreso Internacional Virtual de Educación de la Universidad de Murcia. Recuperado de http://www. acosomoral.org/pdf/E1\%20perfil $\% 20 \mathrm{del} \% 20$ alumno $\% 20$ agresor $\% 20$ en $\% 201 \mathrm{a} \% 20$ escue.pdf

25. Herrera \& Cols. (2002). Adolescencia y comportamiento antisocial. Investigación publicada en Psicothema. 14 (2) 340-343. Recuperado de http:// www.psicothema.com/psicothema.asp? ID =729

26. Ibáñez, M. (1997). Sesgo y consistencia interna de la escala revisada de psicoticismo: Implicaciones conceptuales. Artículo publicado en España en la Revista Electrónica de Motivación y Emoción. Recuperado de http://reme.uji.es/articulos/aorteg8452 21098/texto.html

27. Justicia, F. \& Cols. (2006). Aproximación a un nuevo modelo explicativo del comportamiento antisocial. Revista Electrónica de Investigación Psicoeducativa. 9 (4) 31-150. Recuperado de http://www. investigacion-psicopedagogica.org/revista/ articulos/9/espannol/Art_9_117.pdf

28. Lemos, S. et al. (1992). Salud mental de los adolescentes asturianos. Revista Psicothema 4 (1), 21 -48 .

29. López. \& López (2003). Rasgos de personalidad y conducta antisocial y delictiva. Revista Psicopatología Clínica Legal y Forense, 3 (2) 5 - 19.

30. Martínez, R. \& Cols. (2009). El coeficiente de correlación de los rangos de pearson. Revista Habanera de Ciencias Médicas 8 (2), 17 - 35.

31. Ministerio de Educación del Perú (2006). Convivencia y disciplina escolar democrática. Lima: Editorial Supergráfica E.I.R.L.

32. Montaña de Barragán, C., Acosta \& Gerena (2002). Estudio descriptivo correlacional entre íra y personalidad, a la luz de la teoría de Hans Eysenck. Revista Colombiana de Psicología 2 (11), 15 - 19, Universidad Nacional de Colombia.

33. Morales, F. (2007). El efecto de la impulsividad sobre la agresividad y sus consecuencias en el rendimiento de los adolescentes. Tesis de Licenciatura, Universitat Rovira I Virgili, Cataluña.

34. Mori, P. (2002). Personalidad, Autoconcepto y Percepción del Compromiso Parental: Sus Relaciones con el Rendimiento Académico en Alumnos del Sexto
Grado. Tesis Doctoral, Universidad Nacional Mayor de San Marcos.

35. Moya, J. \& Meseguer (2010). Dimensiones de personalidad, diferencias de género y agresividad. Jornadas de Fomento de la Investigación. Universitat Jaume, Castellón.

36. Muñiz, J. (2000). Teoría clásica de los tests. Madrid: Ediciones Pirámide.

37. Olivero, M. et al. (2008). Violencia escolar en colegios estatales de primaria en el Perú. Revista Peruana de Pediatría, 61 (4) 34 - 41.

38. Oviedo, H \& Arias, A. (2005). Aproximación al uso del coeficiente Alpha de Cronbach. Revista Colombiana de Psiquiatría. 34 (4) 45-65. Recuperado de http://www.scielo.org.co/pdf/rcp/v34n4/v34n4a 09.pdf

39. Ohsako, T. (1997). Violence at School: Global Issues and Interventions. International Bureau of Education. Recuperado de www.ibe.unesco.org/publications/ SalesPublications/salespdf/Ohsako.pdf

40. Olivares, J., Méndez, F. \& Maciá, D. (2003). Tratamientos conductuales en la infancia y adolescencia. Madrid: Psicología Pirámide.

41. Paredes, E. (2002). La enfermedad mental: aspectos psicopatológicos y semiológicos. Lima, Perú: Libro Amigo.

42. Pellegrini, A., Bartini, M. \& Brooks, F. (1999). School aggressors, victims and aggressive victims: Factors related to group affiliation and victimization in early adolescence. Journal of Educational Psychology, 91 (2) 216-224.

43. Piedra, M. (1988). Dimensiones de personalidad y el rendimiento escolar en niños y niñas quinto grado. Tesis de Bachillerato, Pontificia Universidad Católica del Perú.

44. Porrata, J. \& Cols. (2003). Puntuaciones en el cuestionario de personalidad de adolescentes agresivos. Universidad de Puerto Rico. Recuperado de http://jlporrata.net/pdf/PUNTUACIONES CUESTION A R I O PER S ON A L I D A D ADOLESCENTES_AGRESIVOS.pdf

45. Ramírez (2007). Liderazgo desarrollador. Maestría realizada en la Universidad del Valle de México. Recuperada de http://www.lajornadamorelos.com/ index.name $=$ PNphpBB2 $\&$ file $=$ viewtopic $\& p=2604 \&$ POSTNUKESID=8a0639f9bd748ddcc9c250e9c calalfl

46. Rodríguez, N. (2006). Agresión entre pares: Acoso en las aulas. Recuperado de http://misticablog. wordpress.com/2006/05/09/agresión entre paresacoso-en-las-aulas/

47. Schmidt, V. et al. (2010). Modelo psicobiológico de la personalidad de Eysenck: Una historia proyectada hacia el futuro. Revista Internacional de Psicología, Buenos Aires. 11 (2) 32-41. 
48. Serrano, I. (1996). Agresividad infantil. Madrid: Pirámide.

49. Tamayo, J. (1999). Trastornos del control de impulsos. Revista Psicofarmacología-Online. Recuperado de http://psicofarmacologia.info/Timpulsos.html

50. Thunfors, P. \& Cornell, D. (2006). The popularity of middle school aggressors. The Journal of School Violence. Recuperado de http://youthviolence. edschool.virginia.edu/research-publications.htm
51. Teruel, J. (2007). Estrategias para prevenir el agresión entre pares en las aulas. Madrid: Pirámide.

52. Vásquez, C. (2003). Predicción y prevención de la delincuencia juvenil según las teorías del desarrollo social. Revista de Derecho de España. Recuperado de http://mingaonline.uach.cl/scielo.php?script $=$ sci arttext\&pid $=$ S0718-09502003000100008\&lng=es\& nrm=iso 